\title{
Advanced density matrix renormalization group method for nuclear structure calculations
}

\author{
Ö. Legeza, ${ }^{1,{ }^{*}}$ L. Veis,${ }^{1,2, \dagger}$ A. Poves, ${ }^{3, \ddagger}$ and J. Dukelsky ${ }^{4, \S}$ \\ ${ }^{1}$ Strongly Correlated Systems "Lendület” Research group, Wigner Research Centre for Physics, H-1525 Budapest, Hungary \\ ${ }^{2}$ J. Heyrovsky Institute of Physical Chemistry, Academy of Sciences of the Czech Republic (ASCR), CZ-18223 Prague, Czech Republic \\ ${ }^{3}$ Departamento de Física Teórica e IFT-UAM/CSIC, Universidad Autónoma de Madrid, 28049 Madrid, Spain \\ ${ }^{4}$ Instituto de Estructura de la Materia, CSIC, Serrano 123, 28006 Madrid, Spain
}

(Received 6 August 2015; published 9 November 2015)

\begin{abstract}
We present an efficient implementation of the Density Matrix Renormalization Group (DMRG) algorithm that includes an optimal ordering of the proton and neutron orbitals and an efficient expansion of the active space utilizing various concepts of quantum information theory. We first show how this new DMRG methodology could solve a previous $400 \mathrm{keV}$ discrepancy in the ground state energy of ${ }^{56} \mathrm{Ni}$. We then report the first DMRG results in the $p f+g 9 / 2$ shell model space for the ground $0^{+}$and first $2^{+}$states of ${ }^{64} \mathrm{Ge}$ which are benchmarked with reference data obtained from a Monte Carlo shell model. The corresponding correlation structure among the proton and neutron orbitals is determined in terms of two-orbital mutual information. Based on such correlation graphs we propose several further algorithmic improvement possibilities that can be utilized in a new generation of tensor network based algorithms.
\end{abstract}

DOI: 10.1103/PhysRevC.92.051303

PACS number(s): 21.60. $-\mathrm{n}, 75.10 . \mathrm{Jm}, 02.60 .-\mathrm{x}, 27.50 .+\mathrm{e}$

Large-scale shell model calculations in physically sound valence spaces are the prime choice in the nuclear spectroscopy of light and medium mass nuclei. The catch is that the dimensions of the basis (the number of Slater determinants) grow as the product of the two combinatorial numbers made with the number of single-particle states $(n, l, j, m)$ in the valence space and the number of particles, for neutrons and protons. Modern shell model codes can cope with dimensions $O\left(10^{11}\right)[1]$. Going beyond this limit requires clever truncation and extrapolation schemes. In addition, different approximation methods may be implemented. Among them, perhaps the most accurate is the Monte Carlo shell model (MCSM) that stochastically samples the Hilbert space to find the relevant basis states for the description of a particular eigenstate [2]. Several improvements have been added to the original methodology over the years, making the MCSM a robust and accurate technique to study medium-mass nuclei. The most important are the sequential conjugate gradient to select the Slater determinants and the parity and angular momentum projection of the determinants, plus an energy variance extrapolation method [3,4].

Another efficient numerical tool to approximate the exact wave function in a truncated basis is the Density Matrix Renormalization Group (DMRG) method [5] that was earlier introduced in nuclear structures either in the particle-hole basis (phDMRG) [6,7] or in the $j$-coupling scheme (JDMRG) $[7,8]$. Both methods found difficulties in treating systems beyond the limits of an exact diagonalization. However, phDMRG in the $j$-coupling scheme has been successfully applied to the Gamow shell model of weakly bound light nuclei $[9,10]$ due to the weak entanglement between the valence space

\footnotetext{
*legeza.ors@wigner.mta.hu

†libor.veis@jh-inst.cas.cz

${ }^{\ddagger}$ alfredo.poves@uam.es

§dukelsky@iem.cfmac.csic.es
}

and resonances with the discretized continuum. Moreover, a standard implementation of DMRG to nuclear structure found a serious discrepancy of $400 \mathrm{keV}$ for the ${ }^{56} \mathrm{Ni}$ ground state energy in the pf shell [11]. These results hindered further application of the DMRG method to medium-size nuclei. Meanwhile, there have been important efforts made to implement DMRG in quantum chemistry (QC-DMRG) [12] by utilizing various concepts of quantum information theory [13-16]. Nowadays, DMRG is capable of providing the low-lying energy spectrum of complex molecules with great accuracy [17-21] and it has ranked among the standard multireference QC methods.

In this Rapid Communication we will use the new QCDMRG techniques to overcome first the $400 \mathrm{keV}$ discrepancy found in the ${ }^{56} \mathrm{Ni}$ calculations in the $p f$ shell. Afterward, we will show how DMRG can deal with nuclei well beyond the limits of an exact diagonalization by studying ${ }^{64} \mathrm{Ge}$ in the $p f$ and in the enlarged $p f+g 9 / 2$ valence space, and compare our results with benchmark calculations of MCSM. As a byproduct of the method, we will depict the single-site entropy and mutual information of ${ }^{64} \mathrm{Ge}$, shedding new light on the landscape of entanglement and correlations in nuclear structure.

In our DMRG implementation we study the most general Hamiltonian with one- and two-body interaction terms given as

$$
H=\sum_{\alpha} \varepsilon_{\alpha} c_{\alpha}^{\dagger} c_{\alpha}+\frac{1}{2} \sum_{\alpha \beta \gamma \delta} V_{\alpha \beta \gamma \delta} c_{\alpha}^{\dagger} c_{\beta}^{\dagger} c_{\delta} c_{\gamma},
$$

where $c_{\alpha}^{\dagger}$ and $c_{\alpha}$ creates and annihilates a particle with quantum numbers $\alpha=\left(n, l, j, m, \tau_{z}\right)$.

In the so-called $\mathbb{C}^{2}$ representation an orbital can be either empty or occupied, thus the dimension of the local Hilbert space, $\Lambda_{i}$ of a single orbital, $q=\operatorname{dim} \Lambda_{i}$ is 2 . The full Hilbert space of a finite system comprising $N$ orbitals, $\Lambda^{(N)}$, is built from tensor product spaces of local orbital spaces $\Lambda_{i}$, which can be written as $\Lambda^{(N)}=\otimes_{i=1}^{N} \Lambda_{i}$. 
In the DMRG method, quantum correlations are taken into account by an iterative procedure that variationally minimizes the energy of the Hamiltonian given by Eq. (1). The method eventually converges to the full configuration interaction (CI) solution within the selected active orbital space. In the two-site DMRG variant [5,22], $\Lambda^{(N)}$ is approximated by a tensor product space of four tensor spaces defined on an ordered orbital chain, i.e., $\Xi_{\mathrm{DMRG}}^{(N)}=\Xi^{(\mathrm{l})} \otimes \Lambda_{i+1} \otimes \Lambda_{i+2} \otimes \Xi^{(\mathrm{r})}$. The basis of states $\Xi^{(1)}$ comprises $i$ orbitals to the left of the chain $\left(l \equiv\right.$ left) and that of $\Xi^{(\mathrm{r})}$ comprises $N-i-2$ orbitals to the right of the chain ( $r \equiv$ right). These states are determined through a series of unitary transformations based on the singular value decomposition (SVD) theorem by going through the ordered orbital space from left to right and then sweeping back and forth [20,22]. The number of block states, $M_{l}=\operatorname{dim} \Xi^{(1)}$ and $M_{r}=\operatorname{dim} \Xi^{(\mathrm{r})}$, required to achieve sufficient convergence can be regarded as a function of the level of entanglement among the orbitals [13,23]. The maximum number of block states $M_{\max }=\max \left(M_{l}, M_{r}\right)$ required to reach an a priori defined accuracy threshold is inherently determined by truncation error, $\delta \varepsilon_{\mathrm{TR}}$, when the dynamic block state selection (DBSS) approach is used [24]. During the initial sweeps of the DMRG algorithm the accuracy is also affected by the environmental error, $\delta \varepsilon_{\text {sweep }}$. The latter error can be reduced significantly by taking advantage of the CI-based dynamically extended active space (CI-DEAS) procedure $[13,25]$ and using a large number of DMRG sweeps until the energy change between two sweeps is negligible. In the CI-DEAS procedure the active space of orbitals is extended dynamically based on the orbital entropy profile [15]. $M_{\max }$ depends strongly on the orbital ordering along the one-dimensional chain topology of the DMRG method [24,26]. There exist various extrapolation schemes to determine the truncation-free solution [21]. In this work, due to the high level of entanglement of the nuclear wave functions, we carry out the extrapolations as a function of the total number of block states $M$. Using $E(M)=E(M \rightarrow \infty)+X_{1} M^{X_{2}}$ we have estimated the truncation-free solution where $E(M \rightarrow \infty), X_{1}$ and $X_{2}$ are free parameters of our fit [27]. We have performed between 30 to 90 sweeps by requiring the energy change between two sweeps to be below $10^{-4} \mathrm{MeV}$.

The amount of contribution to the total correlation energy of an orbital can be quantified by the single-orbital von Neumann entropy [13], $s_{i}=-\operatorname{Tr} \rho_{i} \ln \rho_{i}$, where $\rho_{i}$ is the reduced density matrix of orbital $i$. The two-orbital von Neumann entropy $s_{i j}$ is constructed similarly using the reduced density matrix $\rho_{i j}$ of a subsystem built from orbitals $i$ and $j$, and the mutual information $I_{i j}=s_{i j}-s_{i}-s_{j}$ describes how orbitals are correlated with each other as they are embedded in the whole system $[14,15,28]$. The orbital ordering is determined by the minimization of the entanglement distance expressed as $I_{\text {dist }}=I_{i j}|i-j|^{\eta}$ where $\eta \geqslant 1$. [14,15]. In this work we used $\eta=2$ in order to carry out the optimization task using concepts of spectral graph theory [29].

Our DMRG code for nuclear structure calculations is composed of two phases. The preprocessing phase in which the orbital ordering and the active space vectors are optimized by calculating the one-orbital entropy and two-orbital mutual information using a fixed small number of block states. The production phase in which an accurate calculation is performed using a large fixed number of block states or the DBSS procedure in order to reach an a priori set accuracy threshold [30]. The preprocessing phase takes only a small fraction of the total computational time.

First we perform the DMRG calculation for the $s d$ nucleus ${ }^{28} \mathrm{Si}$ employing the USD interaction [31]. This system was treated almost a decade ago by the DMRG method [11]. By keeping fixed $M=1024$ block states we have reproduced the exact value of the $0^{+}$ground state energy and excitation energy of the first $2^{+}$state. Using the quantum information entropy based ordering optimization, the CI-DEAS procedure, and the DBSS approach with $M_{\min }=256$ and $\delta \varepsilon_{\mathrm{TR}}=10^{-3}-10^{-6}$ we determined the ground state energy within the a priori set accuracy threshold. This drastic improvement clearly demonstrates the importance of the quantum entropy based optimization procedures.

Next we consider the nucleus ${ }^{56} \mathrm{Ni}$ with the KB3 interaction $[32,33]$. The exact results in $p f$ valence space were obtained by the code ANTOINE [1]. The basis dimension in the $m$-scheme for $J_{z}=0$ is $1.1 \times 10^{9}$. In Fig. 1(a) the DMRG ground state energy for ${ }^{56} \mathrm{Ni}$ is shown as a function of $1 / M$. For small $M$ values a downward curvature governs the behavior of the scaling function (indicated by the dotted lines); while for large enough $M$ values, an inflection point is reached and an upward curvature becomes apparent. Our lowest variational energy obtained with $M=4096$ states is $E_{\mathrm{GS}}^{(\mathrm{Ni})}(M=4096)=$ $-78.451 \mathrm{MeV}$, thus the error compared to the exact value, $E_{\text {exact }}=-78.465 \mathrm{MeV}$, is $1.4 \times 10^{-2} \mathrm{MeV}$.

Using the fit function defined above we have estimated the ground state energy in the $M \rightarrow \infty$ limit as $E_{\mathrm{GS}}^{(\mathrm{Ni})}(M \rightarrow$ $\infty)=-78.463(4) \mathrm{MeV}$. The deviation from the exact ground state energy is in the order of a few keV's. Therefore, using entropy based optimized DMRG methodology we could solve

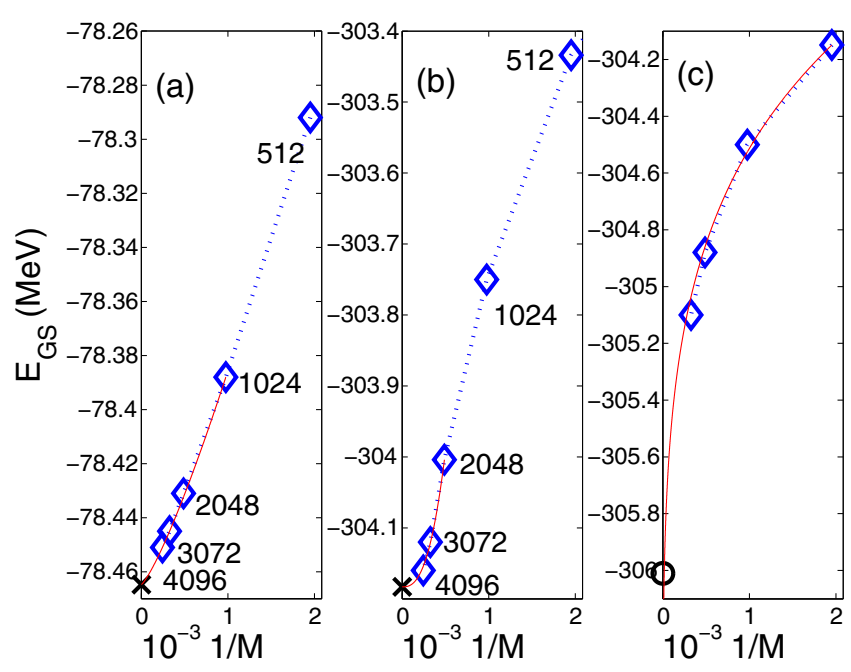

FIG. 1. (Color online) DMRG ground state energy for ${ }^{56} \mathrm{Ni}$ and ${ }^{64} \mathrm{Ge}$ in the $p f$ shell and ${ }^{64} \mathrm{Ge}$ in the $p f+g 9 / 2$ valence space as functions of $1 / M$; diamonds (DMRG), crosses (exact diagonalization), circle (MCSM). The solid lines are the fits that produce our extrapolated value, while the dotted lines joining the points not used in the fit are just to guide the eye. 
the previous $400 \mathrm{keV}$ discrepancy in the ground state energy of ${ }^{56} \mathrm{Ni}$ reported in Ref. [11].

Next we study ${ }^{64} \mathrm{Ge}$ in the $p f$ valence space for which we have used the GXPF1A interaction [34,35]. The DMRG results for the $p f$ valence space are displayed in Fig. 1(b). Our lowest variational energy obtained with $M=4096$ states is $E_{\mathrm{GS}}^{(\mathrm{Ge})}(M=4096)=-304.163 \mathrm{MeV}$ which extrapolates to $E_{\mathrm{GS}}^{(\mathrm{Ge})}(M \rightarrow \infty)=-304.182(3) \mathrm{MeV}$ while the exact energy obtained with the code ANTOINE is $E_{\text {exact }}=-304.183 \mathrm{MeV}$. Therefore, the error is again in the keV range.

Being confident with the results obtained in the $p f$ shell, we proceed to explore the ability of DMRG to describe accurately the structure of nuclei in valence spaces that exceed the limits of an exact diagonalization. With this in mind, we study ${ }^{64} \mathrm{Ge}$ in the extended space $p f+g 9 / 2$ (dimension in $m$-scheme $1.7 \times 10^{14}$ ) which was already considered using moment methods [35] and the MCSM [4,36]. In this case the GXPF1A interaction has to be completed with the matrix elements between the $p f$-shell orbits and the $g 9 / 2$ obtained in a standard $G$-matrix calculation. [37] In addition, the spurious center of mass contamination is treated with Lawson's ansatz [38]. Within this extended valence space we found a significantly slower convergence rate as a function of $M$ as can be seen in Fig. 1(c). Even using up to $M=3072$ block states the inflection could not be reached, thus the upward curvature did not become apparent yet. Therefore, the estimated energy using our fit function overshoots the reference MCSM energy $\left(E_{\mathrm{MCSM}}=\right.$ -306.066) $\mathrm{MeV}$ and can provide only a lower bound which we found to be $E_{\mathrm{GS}}^{(\mathrm{Ge})}(M \rightarrow \infty)=-307.4 \mathrm{MeV}$. An upper bound can be estimated using a second-order polynomial fit giving $E_{\mathrm{GS}}^{(\mathrm{Ge})}(M \rightarrow \infty)=-305.5 \mathrm{MeV}$. The extrapolated MCSM reference energy lies within the two bounds given above. Due to the slow scaling of the energy as a function of $1 / M$, significantly more block states are needed to provide a reliable extrapolation.

Besides optimization procedures the entanglement analysis is also very important to obtain physical information encoded in the wave functions [16,28,30,39]. The single-orbital entropy and two-orbital mutual information obtained with DMRG for ${ }^{64} \mathrm{Ge}$ are shown in Fig. 2 for an ordering with protons situated on the left side and neutrons on the right side of the chain. For clearness of the physical picture we have selected the spherical Hartree-Fock ordering of the single-particle orbits instead of the optimal ordering used in the DBSS procedure.

The single-orbital entropy profiles reflect the strong configuration mixing in the wave function. The magnetic states belonging to the same orbital have the same entropy within the numerical accuracy. The small differences in the single-orbital entropy within a given $j$-shell are due to the fact that, even though in the present application we have controlled the number of protons and neutrons and the $J_{z}$ quantum number, we do not enforce total angular momentum conservation. Such differences get smaller with lowering the accuracy threshold or increasing the number of block states leading to a better conserved $J^{2}$ value. Alternatively it could be possible to incorporate angular momentum conservation in the code along the lines of Refs. [7-10]. The entropies of the $p 1 / 2$ and $p 3 / 2$
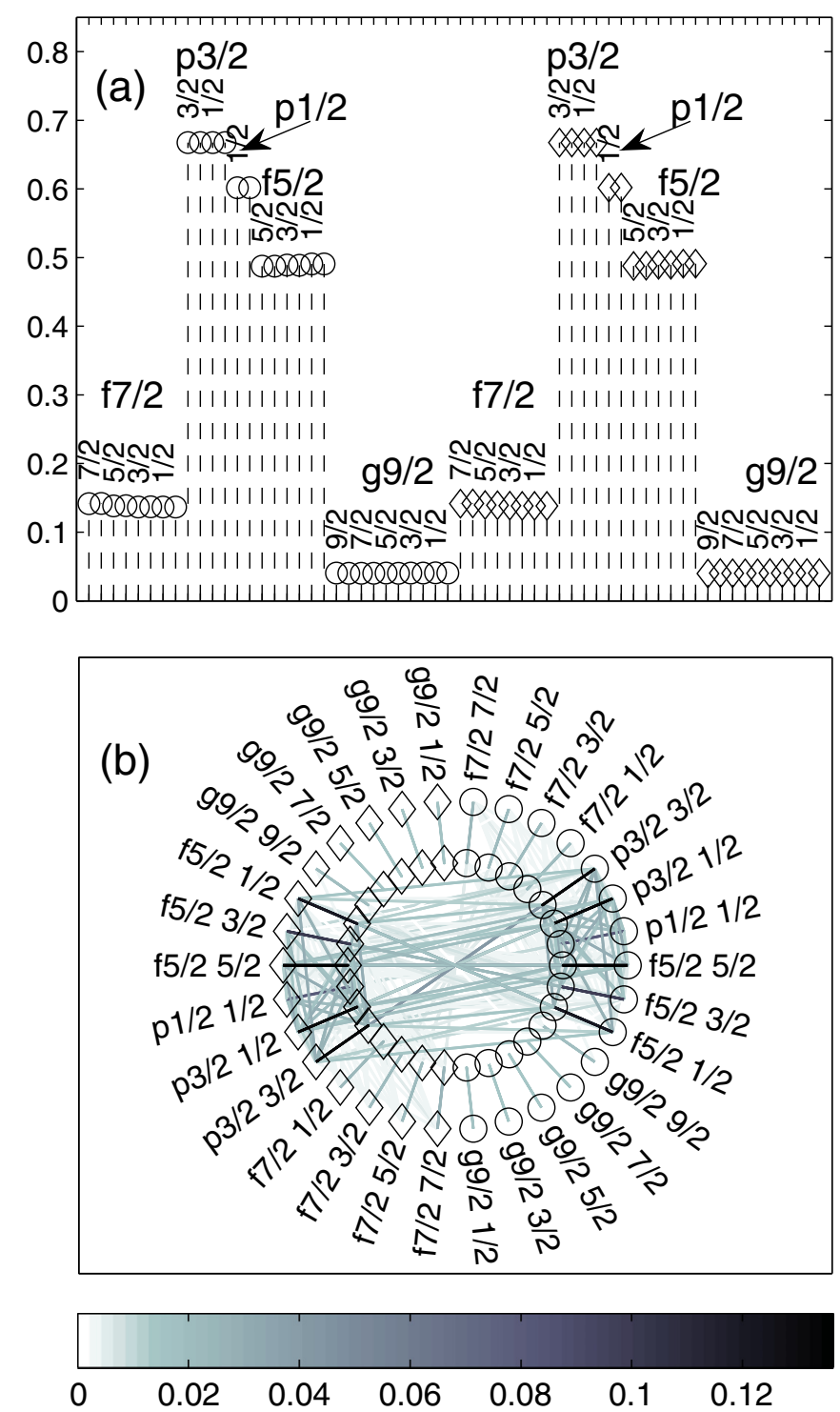

FIG. 2. (Color online) Single-site entropy (a) and mutual information (b) obtained with DMRG for ${ }^{64} \mathrm{Ge}$ in the $p f+g 9 / 2$ shell. The mutual information matrix elements are shown on a ladder topology with time-reversed pairs in the rungs. Circles and diamonds label proton and neutron orbitals, respectively, and sites are denoted by $l, j, m$ with $+m$ outside the ladder and $-m$ inside.

orbitals are very close to the upper limit $\ln 2=0.693$ which corresponds to the maximally mixed state. Orbitals with the largest entropies contribute the most to the correlation energy, thus their accurate treatment is mandatory. As expected, the entropy profiles show that the $p 1 / 2, p 3 / 2$, and $f 5 / 2$ orbits play a dominant role in the physics of the system, whereas the presence of the $f 7 / 2$ orbit is non-negligible and that of the $g 9 / 2$ is minor. Notice that these three highly entangled orbits close a pseudo-SU(3) symmetry which enhances the quadrupole-quadrupole correlations, as will be apparent in the mutual information diagram.

The two-orbital mutual information shows how orbitals are correlated with each other. The rungs of the ladder display 
the degree of entanglement between time reversed states of like particles. The fact that the mutual information is approximately equal for the $p 1 / 2, p 3 / 2$, and $f 5 / 2$ orbits, and independent of their $j_{z}$ projections, is consistent with the presence of a strong $\mathrm{T}=1$ proton-proton and neutron-neutron pairing coherence. The same coherence, though significantly less intense, is seen in the $f 7 / 2$ and the $g 9 / 2$. Proton-neutron $\mathrm{T}=1$ pairing correlations could also be seen between time reversed and charge conjugated states for the $p 1 / 2, p 3 / 2$, and $f 5 / 2$ orbits. We could also see significant entanglement between proton-neutron maximally aligned states for the $p 3 / 2$ and $f 5 / 2$ orbits, which could be related to $J=2 j_{z}$ pairing and/or quadrupole-quadrupole in the $\mathrm{T}=0$ channel. Similarly, quadrupole correlations can be observed inside the ladder (proton-proton and neutron-neutron) as well as those connecting opposite sites (proton-neutron) of the ladder for the $p 1 / 2, p 3 / 2$, and $f 5 / 2$ orbits.

Let us consider now the excitation energies of the $2^{+}$states. It is worth remarking that even in the case when the ground state energy could not be determined with the desired accuracy, the energy difference between two states can be obtained more accurately due to cancellation of errors by targeting the two lowest lying eigenstates simultaneously. The exact value of the $2^{+}$excitation energy for ${ }^{28} \mathrm{Si}$ can be reproduced with $M=1024$ block states. For ${ }^{56} \mathrm{Ni}$, using fixed $M=2048$ block states, we obtain $\Delta E=5.218 \mathrm{MeV}$ to be compared with the exact value $5.125 \mathrm{MeV}$, with an absolute error of $93 \mathrm{keV}$. For ${ }^{64} \mathrm{Ge}$ in the $p f$ shell we get $\Delta E=0.922 \mathrm{MeV}$ compared with the exact value $0.906 \mathrm{MeV}$, the absolute error being now $16 \mathrm{keV}$. Using the fitting procedure to extrapolate the $2^{+}$ excitation energies to $M=\infty$ we obtain 5.121(3) and 0.907(2) $\mathrm{MeV}$ for ${ }^{56} \mathrm{Ni}$ and ${ }^{64} \mathrm{Ge}$. Indeed the agreement is excellent.

When the $p f+g 9 / 2$ valence space is considered we found a slower convergence rate as a function of $1 / M$ due to the more complex entanglement structure in the system. Figures 3(a) and 3 (b) show the $2^{+}$excitation energies of ${ }^{64} \mathrm{Ge}$ as a function of $1 / M$ for the $p f$ shell and $p f+g 9 / 2$ shell, respectively. The solid lines represent the fitted values. In the latter case the exact solution is not available, thus the extrapolated MCSM energies [36], $\Delta E=0.919$ (without reordering) or $\Delta E=0.890$ (with reordering), can be taken as benchmark references and are indicated by the blue diamond symbol. Our extrapolated excitation energy $\Delta E=0.90(2)$ is in good agreement with the MCSM result.

In this work we have demonstrated that the DMRG method including various novel optimization algorithms based on quantum information theory can be applied efficiently to nuclear structure calculations for medium-mass nuclei in extended valence spaces with dimensions exceeding the limits of an exact diagonalization. The DMRG results for the ground state energy of ${ }^{64} \mathrm{Ge}$ in the $p f+g 9 / 2$ valence space could be further improved in order to obtain a reliable estimate for the truncation-free limit. However, the excitation energy of the first $2^{+}$is in excellent agreement with MCSM benchmark results. By calculating the single-orbital and two-orbital mutual information we have determined the correlation structure
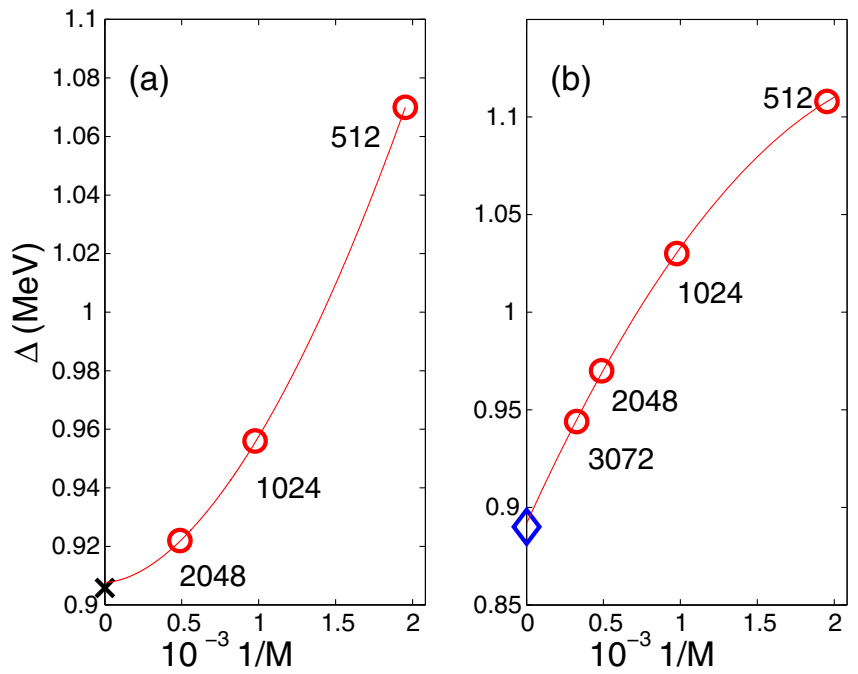

FIG. 3. (Color online) Energy gaps between the 0 and $2^{+}$states for ${ }^{64} \mathrm{Ge}$ with $p f$ (a) and $p f+g 9 / 2$ (b) valence space as a function of $1 / M$; circles (DMRG), cross (exact diagonalization), diamond (MCSM). The solid lines are our fits.

among the orbitals. These entanglement graphs, based on recent developments in QC applications [20], constitute a novel and highly precise tool to picture wave-function correlation properties. The analysis of these entanglement graphs suggests new alternatives for efficient truncation methods that can be developed on general grounds. A straightforward extension of the DMRG algorithm could be the use of local tensors with $\Lambda_{i}$ of dimension $q=4$, but unlike the QC implementations, the states to include in this tensor should be time-reversed pairs in order to optimize the treatment of nuclear pairing correlations. As a final remark, the use of tree-tensor network state (TTNS), which is a recent development in quantum information theory [40-42], could tackle the problem of having equal single entropy values for each orbital group that makes inefficient a sequential treatment of these states within an ordered chain. Within this scheme, several orbitals with equally large entropy values could form the central shells of the TTNS network. These new developments could certainly open the field of medium to heavy nuclei to highly precise spectroscopic calculations.

\section{ACKNOWLEDGMENTS}

We thank the hospitality of NORDITA during the workshop "Computational Challenges in Nuclear and Many-Body Physics" when this project started. We acknowledge the invaluable help of M. Horoi and T. Papenbrock in the initial part of the project. This work was supported in part by the Hungarian Research Fund (OTKA) through Grants No. K100908 and No. NN110360, and by the Spanish Ministry of Economy and Competitiveness through Grants No. FIS201234479, No. FPA2014-57196, and No. SEV2012-0249. 
[1] E. Caurier, G. Martínez-Pinedo, F. Nowacki, A. Poves, and A. P. Zuker, Rev. Mod. Phys. 77, 427 (2005).

[2] M. Honma, T. Mizusaki, and T. Otsuka, Phys. Rev. Lett. 75, 1284 (1995).

[3] T. Mizusaki and M. Imada, Phys. Rev. C 65, 064319 (2002).

[4] N. Shimizu, Y. Utsuno, T. Mizusaki, T. Otsuka, T. Abe, and M. Honma, Phys. Rev. C 82, 061305(R) (2010).

[5] S. R. White, Phys. Rev. Lett. 69, 2863 (1992).

[6] J. Dukelsky, S. Pittel, S. S. Dimitrova, and M. V. Stoitsov, Phys. Rev. C 65, 054319 (2002).

[7] J. Dukelsky and S. Pittel, Rep. Prog. Phys. 67, 513 (2004).

[8] B. Thakur, S. Pittel, and N. Sandulescu, Phys. Rev. C 78, 041303(R) (2008).

[9] J. Rotureau, N. Michel, W. Nazarewicz, M. Ploszajczak, and J. Dukelsky, Phys. Rev. Lett. 97, 110603 (2006).

[10] J. Rotureau, N. Michel, W. Nazarewicz, M. Ploszajczak, and J. Dukelsky, Phys. Rev. C 79, 014304 (2009).

[11] T. Papenbrock and D. J. Dean, J. Phys. G 31, S1377 (2005).

[12] S. R. White and R. L. Martin, J. Chem. Phys. 110, 4127 (1999).

[13] Ö. Legeza and J. Sólyom, Phys. Rev. B 68, 195116 (2003); 70, 205118 (2004).

[14] J. Rissler, R. M. Noack, and S. R. White, Chem. Phys. 323, 519 (2006).

[15] G. Barcza, Ö. Legeza, K. H. Marti, and M. Reiher, Phys. Rev. A 83, 012508 (2011).

[16] Y. Kurashige, G. K.-L. Chan, and T. Yanai, Nat. Chem. 5, 660 (2013).

[17] Ö. Legeza, R. Noack, J. Sólyom, and L. Tincani, in Computational Many-Particle Physics, edited by H. Fehske, R. Schneider, and A. Weisse, Lecture Notes in Physics 739, (Springer, Berlin, 2008), p. 653.

[18] K. H. Marti and M. Reiher, Z. Phys. Chem. 224, 583 (2010).

[19] G. K.-L. Chan and S. Sharma, Annu. Rev. Phys. Chem. 62, 465 (2011).

[20] Sz. Szalay, M. Pfeffer, V. Murg, G. Barcza, F. Verstraete, R. Schneider, and Ö. Legeza, Int. J. Quantum Chem. 115, 1342 (2015).

[21] S. Wouters and D. Van Neck, Eur. Phys. J. D 68, 272 (2014).

[22] U. Schollwöck, Rev. Mod. Phys. 77, 259 (2005).
[23] G. Vidal, J. I. Latorre, E. Rico, and A. Kitaev, Phys. Rev. Lett. 90, 227902 (2003).

[24] Ö. Legeza, J. Röder, and B. A. Hess, Phys. Rev. B 67, 125114 (2003).

[25] Ö. Legeza, and J. Sólyom, in International Workshop on "Recent Progress and Prospects in Density-Matrix Renormalization", Lorentz Center, Leiden University, The Netherlands, 2004, http://www.itp.unihannover.de/ jeckelm/dmrg/workshop/ proceedings/ (unpublished).

[26] G. Moritz, B. A. Hess, and M. Reiher, J. Chem. Phys. 122, 024107 (2005).

[27] G. Barcza, O. Legeza, R. M. Noack, and J. Sólyom, Phys. Rev. B 86, 075133 (2012).

[28] K. Boguslawski, P. Tecmer, G. Barcza, Ö. Legeza, and M. Reiher, J. Chem. Theory Comput. 9, 2959 (2013).

[29] J. E. Atkins, E. G. Boman, and B. Hendrickson, SIAM J. Comput. 28, 297 (1998).

[30] E. Fertitta, B. Paulus, G. Barcza, and Ö. Legeza, Phys. Rev. B 90, 245129 (2014).

[31] B. Brown and B. H. Wildenthal, Ann. Rev. Nucl. Part. Sci. 38, 29 (1988).

[32] T. T. S. Kuo and G. E. Brown, Nucl. Phys. A 114, 241 (1968).

[33] A. Poves and A. P. Zuker, Phys. Rep. 70, 235 (1981).

[34] M. Honma, T. Otsuka, B. A. Brown, and T. Mizusaki, Phys. Rev. C 69, 034335 (2004).

[35] R. A. Sen'kov and M. Horoi, Phys. Rev. C 82, 024304 (2010).

[36] N. Shimizu, Y. Utsuno, T. Mizusaki, M. Honma, Y. Tsunoda, and T. Otsuka, Phys. Rev. C 85, 054301 (2012).

[37] M. Honma (private communication).

[38] D. Gloeckner and R. Lawson, Phys. Lett. B 53, 313 (1974).

[39] K. Boguslawski, P. Tecmer, Ö. Legeza, and M. Reiher, J. Phys. Chem. Lett. 3, 3129 (2012).

[40] V. Murg, F. Verstraete, Ö. Legeza, and R. M. Noack, Phys. Rev. B 82, 205105 (2010).

[41] N. Nakatani and G. K.-L. Chan, J. Chem. Phys. 138, 134113 (2013).

[42] V. Murg, F. Verstraete, R. Schneider, P. Nagy, and Ö. Legeza, J. Chem. Theory Comput. 11, 1027 (2015). 